\title{
Magnetic resonance imaging for diagnosis of bipartite patella: usefulness and relationship with symptoms
}

\author{
Tuba Akdag ${ }^{A, B, C, D, E, F}$, Esra S. Guldogan ${ }^{A, B, D, E, F}$, Huseyin Coskun ${ }^{A, B, C, D, E}$, Aynur Turan ${ }^{A, B, C, D, E}$, Baki Hekimoglu ${ }^{C, D, E, F}$
}

University of Health Sciences, Diskapi Yildirim Beyazit Training and Research Hospital, Ankara, Turkey

\section{Abstract}

Purpose: Bipartite patella is a rare developmental variation of the knee cap. We aimed to identify the magnetic resonance imaging (MRI) features of bipartite patella and evaluate the association with clinical symptoms.

Material and methods: MRI exams of 61 patients with bipartite variant were evaluated for types of bipartite patella, oedema around the synchondrosis, bipartite fragment height $(\mathrm{FH})$, distance between the fragment and the patella (FPD), and signal characteristics within the synchondrosis. The study was designed with two observers in order to achieve intra- and interobserver compliance. Any other major knee pathologies that can cause pain were also recorded.

Results: Of the 61 participants the average age was $40.1 \pm 14.3$ years, 44 were males, and 17 were females. Fifty-nine of the bipartite fragments were located at the superolateral quadrant of the patella. There was oedema at the bipartite area in 35 patients. Ten of these patients had no major MRI diagnosis other than oedema, and they were classified as the symptomatic group. The age of the patients in the symptomatic group was statistically lower than in the asymptomatic group $(p<0.05)$. There was no statistically significant difference between the groups in terms of male and female distributions, signal types across the synchondrosis, and FH or FPD measurements $(p>0.05)$. High concordance correlation coefficients were observed on measurements

Conclusions: MRI of the knee is highly accurate in evaluation of bipartite patella. To our knowledge; a detailed MRI analysis, like in our study, has not previously been performed, and our report is unique in showing that the symptomatic occurrence of bipartite patella is statistically higher in young patients.

Key words: patella, bipartite, magnetic resonance, oedema.

\section{Introduction}

The patella is the largest sesamoid bone of the body, which is located in the anterior part of the knee joint. It is the attachment point of quadriceps and patellar tendon and supports the extensor mechanism of the knee [1]. It begins to develop from mesenchymal cells in embryonic life and first ossifies in the fifth year of life. By formation and unification of ossification centres during the adolescent period, the patella reaches its mature formation in adulthood. It is thought that a breakdown at a certain level of merging in patellar ossification centres may cause forma- tion of the bipartite variant [2]. Possible other aetiological factors include older repetitive knee traumas and congenital growth defects $[3,4]$.

Bipartite patella was first reported as a case by Gruber in 1883, in which the patella was made up of two fragments: a large sub-inner and a small sub-outer [5]. Since then, many studies have found different data on its prevalence, classification, and clinical importance. The literature mentions different prevalence rates ranging from $0.8 \%$ to $2 \%$, and it is seen three times more in males than in females [6,7]. Radiological diagnosis can be made by radiograph, computed tomography (CT), or magnetic

\section{Correspondence address:}

Dr. Tuba Akdag, University of Health Sciences, Diskapi Yildirim Beyazit Training and Research Hospital, Ankara, Turkey, e-mail: tubakayi@gmail.com

Authors' contribution:

A Study design · B Data collection · C Statistical analysis · D Data interpretation · E Manuscript preparation · F Literature search · G Funds collection 
resonance imaging (MRI). Radiograph and CT provide insight into the anatomical structure of the variant only. MRI can be successful in explaining the symptoms of patients by detecting bone marrow oedema. Initial treatment of symptomatic bipartite patella is done conservatively. Most of the cases improve with resting and restricting sports activities. Non-steroidal anti-inflammatory drugs are used as the first choice in medical treatment. Surgical treatment is recommended for those who do not respond to conservative treatment.

There are few studies in the literature describing MRI findings in bipartite patella. Here we evaluated the prevalence of bipartite patella and perform a detailed MRI analysis of this rare kneecap variant. We believe that the results of this study will guide future studies.

\section{Material and methods}

\section{Patients}

This study was retrospective, it was performed in accordance with the ethical guidelines of the Helsinki Declaration, and it was approved by the Ethics Committee of our centre. Knee MRI reports of 85,342 patients undergoing MRI in our department between 2014-2018 were screened on the database of the operating system using the keyword 'bipartite'. Patients with a history of trauma and a tripartite or multipartite appearance in the fragment were excluded from the study. Three patients were excluded: two of them had a history of trauma, and one patient had a tripartite variant.

\section{Magnetic resonance imaging protocol}

All images were obtained from 1.5 T MRI devices belonging to the hospital radiology unit. The standard knee protocol using a knee coil included the following sequences: sagittal proton density turbo spin echo (TSE) with fat saturation, sagittal T2 gradient echo (GRE), coronal T1 TSE, coronal proton density TSE with fat saturation, and axial proton density TSE with fat saturation. The following sequences were utilised: sagittal proton density TSE with fat saturation, field of view (FOV): $16 \mathrm{~cm}$, matrix: $240 \times 320$, slice thickness: $3 \mathrm{~mm}$, time to repetition (TR): 2620 time to echo (TE): 40 . Sagittal T2 gradient echo FOV: $16 \mathrm{~cm}$, matrix: $240 \times 320$, slice thickness: $3 \mathrm{~mm}$, TR: 322 TE: 14 . Coronal T1 turbo spin echo, FOV: $16 \mathrm{~cm}$, matrix: $240 \times 320$, slice thickness: $3 \mathrm{~mm}$, TR: 464 , TE: 9.4. Coronal proton density TSE with fat saturation, FOV: $16 \mathrm{~cm}$, matrix: $240 \times 320$, slice thickness: $3 \mathrm{~mm}$, TR: 2740, TE: 21 . Axial proton density TSE with fat saturation, FOV: $16 \mathrm{~cm}$, matrix: $240 \times 320$, slice thickness: $3 \mathrm{~mm}$, TR: 2870, TE: 30 .

The demographic characteristics of 61 patients who were included to the study, such as age and gender, were recorded. MRI analyses were performed by examiners who had at least five years of experience in the field of musculoskeletal radiology.

The evaluation started with the determination of the bipartite patella type, classified as I, II, and III. Type I was defined as a fragment located at the inferior pole; type II, at the lateral margin; and type III, at the superolateral pole. Oedema at bipartite fragment and on the adjacent patella were evaluated and divided into two groups as "oedema present" or "oedema absent". The signals within the synchondrosis were analysed and divided into three groups and recorded as cartilage signal, fluid signal, and fibrous signal. The presence of similar signal properties with patellofemoral cartilage were defined as cartilage signals (Figure 1). The presence of similar signal properties with knee joint fluid were defined as fluid signal (Figure 2). The presence of a hypointense signal in all sequences was defined as a fibrous signal (Figure 3). Major potential causes of pain on knee MRI, such as osteoarthritis, cruciate ligament and meniscus pathologies, soft tissue, and cartilage and extensor mechanism injuries, were noted.
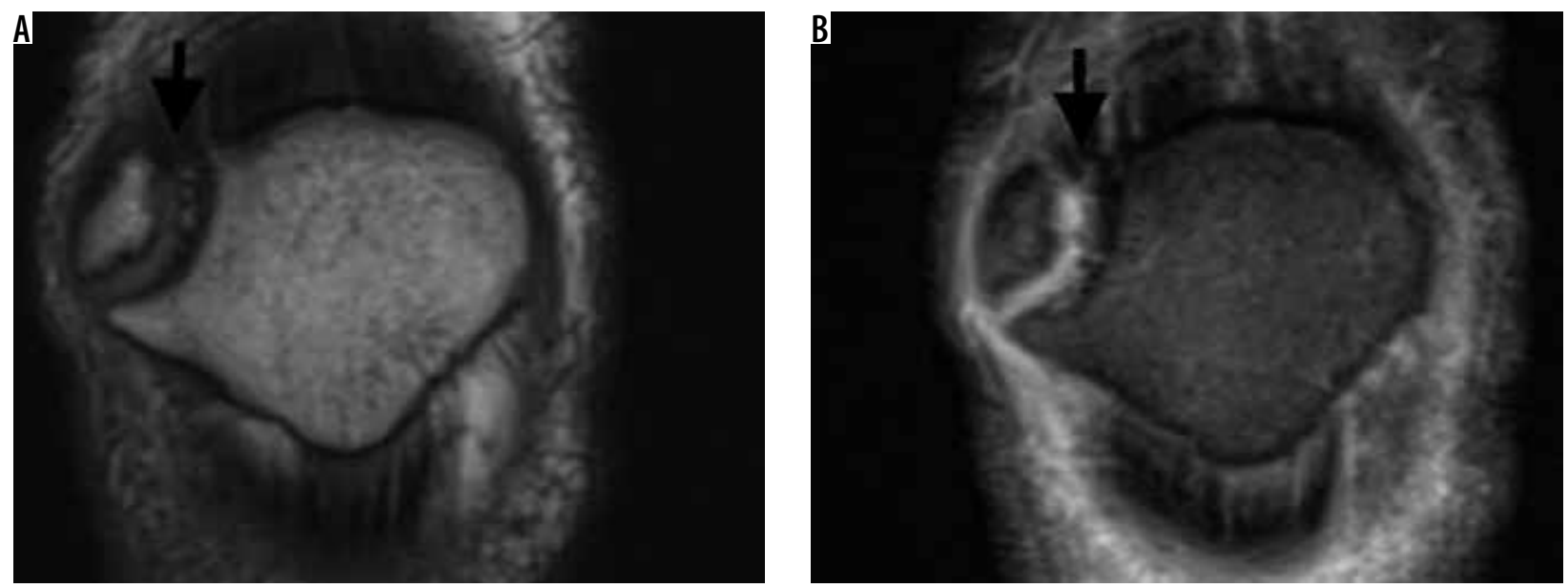

Figure 1. A) Coronal T1W magnetic resonance image confirms the presence of a bipartite fragment at the superolateral pole of the patella. Also note the cartilage signal across the synchondrosis (arrow). B) Coronal T2W image confirms the presence of cartilage signal across the synchondrosis (arrow) 

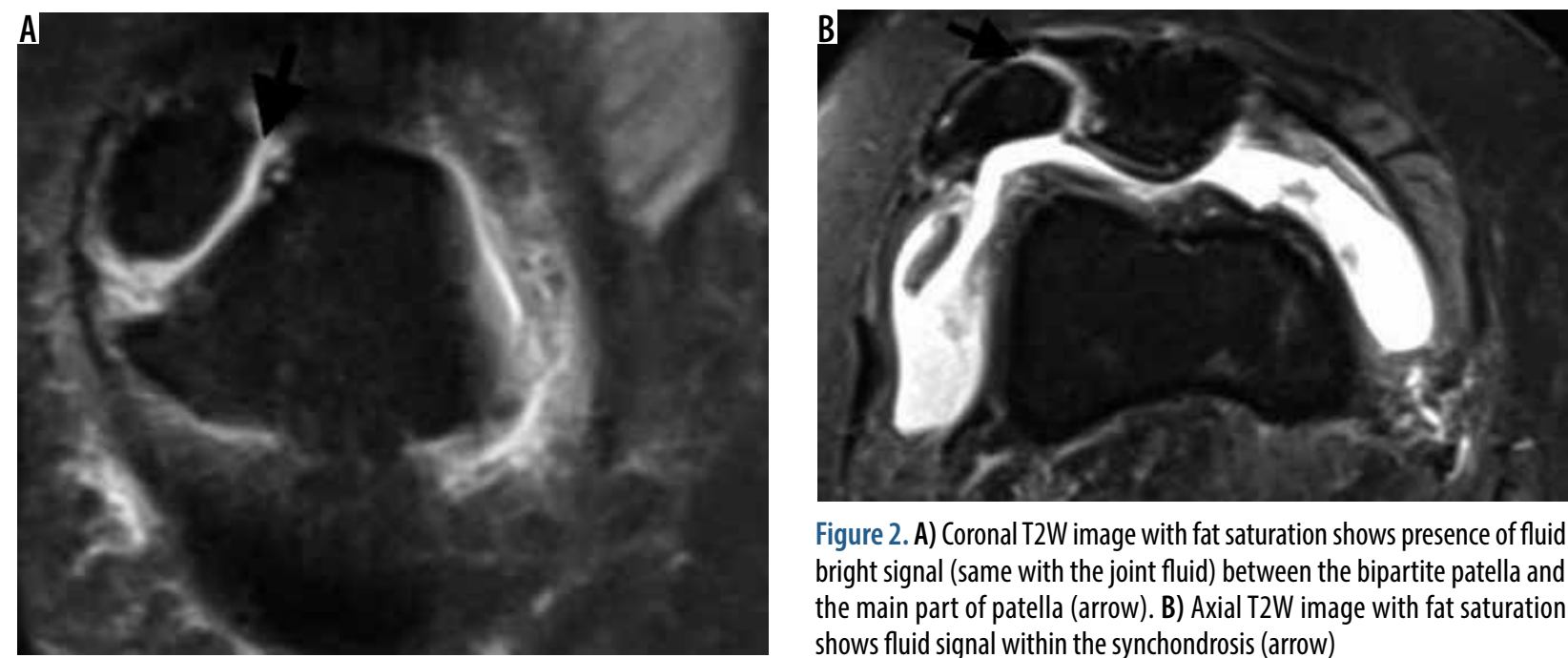

Figure 2. A) Coronal T2W image with fat saturation shows presence of fluid bright signal (same with the joint fluid) between the bipartite patella and the main part of patella (arrow). B) Axial T2W image with fat saturation shows fluid signal within the synchondrosis (arrow)
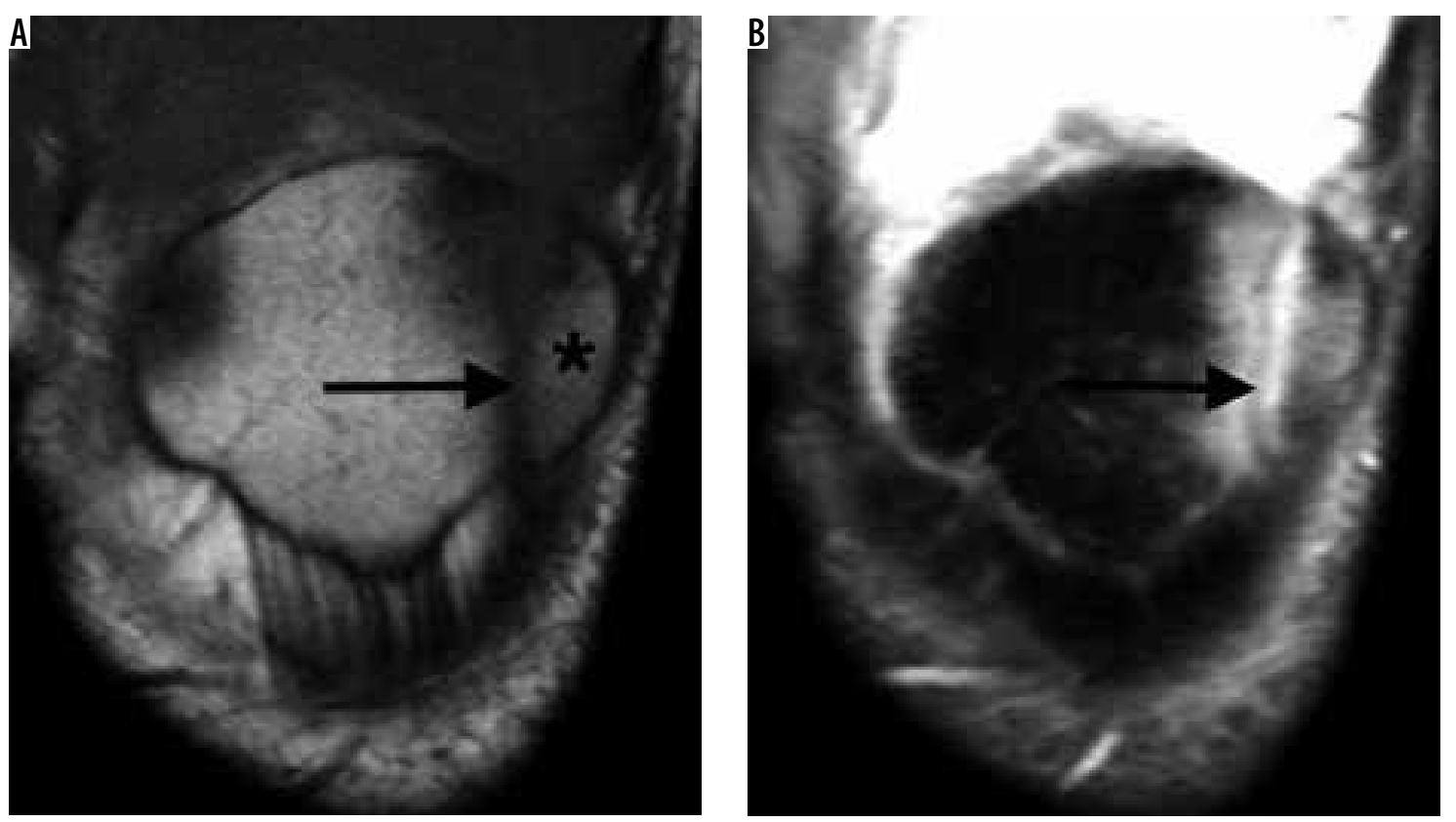

Figure 3. A) Coronal T1-weighted image shows the presence of a lateral pole Type 2 bipartite patella (black star) and hypointense signal between the bipartite fragment and patellar body (arrow). B) Coronal T2-weighted image with fat saturation confirms the fibrous union present between the patella and its bipartite fragment (arrow). Note the presence of oedema around the synchondrosis

Thereafter, in order to form a consensus, the data were reviewed together by the radiologists who evaluated the images.

In addition, the height of the bipartite fragment (fragment height - FH) and the measurement of the distance between the fragment and the patella (fragment-patella distance - FPD) were evaluated. The measurements were performed simultaneously on the same day by two different musculoskeletal radiologists on different computers for inter-observer differences and were repeated for the same observer after a two-week interval for intra-observer differences. FH was measured from the most superior margin to the distal anterior tip by excluding osteophytes from the sagittal T2A PD images (Figure 4A). The highest visible section of the fragment was selected for analysis. The measurement of FPD was performed from the sagit- tal axis, where all the synchondrosis was seen. If no such axis was found, these measurements were included in the study by determination for the same cross-sectional features in axial T2A PD images (Figure 4B). Intraobserver agreements for $\mathrm{FH}$ and FPD were good to excellent ( $\kappa=0.78,0.88$, and 0.77 , respectively), as were interobserver agreements $(\kappa=0.69,0.78$, and 0.79 , respectively).

Finally, to detect the real prevalence of bipartite patella, the coronal T1A images of knee MRIs that performed in the last month were evaluated by another general radiologist who did not participate in the first part of the study. In total, 695 knee MRI images were evaluated. Patients with a history of trauma and a tripartite or multipartite appearance were excluded from the group. Thus, the prevalence of bipartite patella was determined in a large series of patients. 

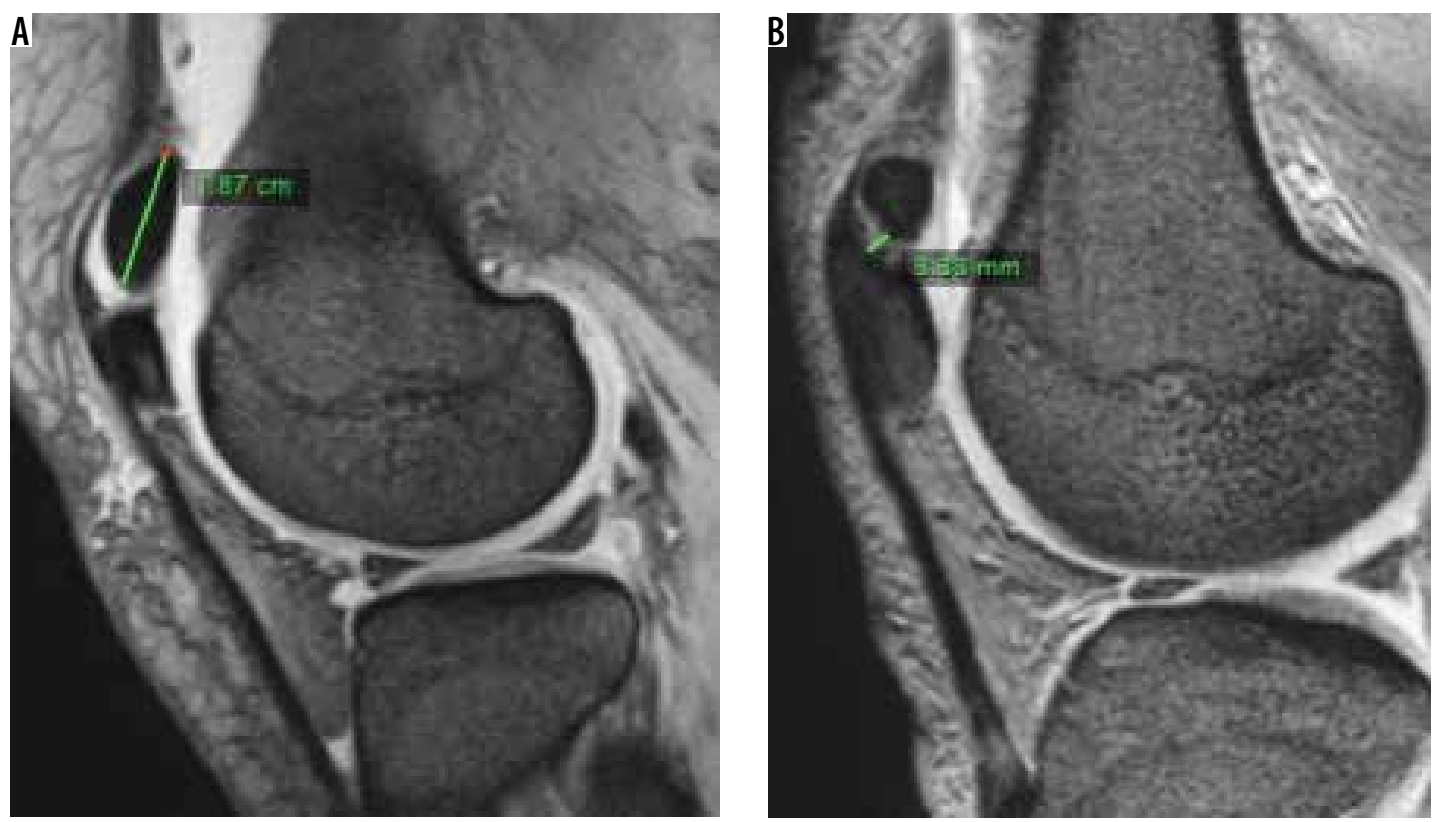

Figure 4. A) Sagittal T2W PD fast spin echo magnetic resonance imaging (MRI) demonstrates the measurement of bipartite fragment height, which is measured on midsagittal images of the fragment from the superior margin to the distal anterior tip. B) Sagittal T2W PD fast spin echo MRI shows the measurement of the distance between the fragment and the patella. Selected cross-section in which the synchondrosis can be seen in a continuous way

\section{Statistical analysis}

Data analysis was performed by using IBM SPSS Statistics version 17.0 software (IBM Corporation, Armonk, NY, USA). Whether the distributions of continuous variables were normally distributed or not was determined by Kolmogorov-Smirnov test. Homogeneity of variance assumption was examined by Levene test. Descriptive statistics for the continuous variables were presented as mean \pm SD or median $\left(25^{\text {th }}-75^{\text {th }}\right)$ percentile, where appropriate. Number of cases and the percentage were used for the categorical data. Intraclass correlation coefficients and

Table 1. Demographic and magnetic resonance imaging characteristics

\begin{tabular}{|l|c|}
\hline Age (years) & $n=61$ \\
\hline Range of age (years) & $40.1 \pm 14.3$ \\
\hline Gender & $14-70$ \\
\hline Male & \\
\hline Female & $44(72.1 \%)$ \\
\hline Classification of bipartite patella & $17(27.9 \%)$ \\
\hline Type I & \\
\hline Type II & None \\
\hline Type III & $2(3.3 \%)$ \\
\hline Oedema around bipartite variant & $59(96.7 \%)$ \\
\hline Cartilage signal & $35(57.4 \%)$ \\
\hline Fibrous signal & $26(42.6 \%)$ \\
\hline Fluid signal & $8(13.1 \%)$ \\
\hline Other knee pathologies & $27(44.2 \%)$ \\
\hline
\end{tabular}

95\% confidence intervals were calculated for determining both intra- and inter-observer agreement levels for fragment height and distance between patella and fragment measurements. The mean differences between groups were compared by Student's $t$-test; and the Mann-Whitney $U$ test was applied for the comparisons of non-normally distributed data. Categorical data were analysed by Fisher's exact test. Degrees of associations between continuous variables were evaluated by Spearman's Rank correlation analyses. A $p$ value less than 0.05 was considered statistically significant.

The intra-observer reproducibility for assessing the $\mathrm{FH}$ and FPD, depicted as intraclass correlation coefficients, was 0.9 (95\% CI: 0.77 to 0.95 ) and 0.98 (95\% CI: 0.96 to 0.99 ), respectively. The reproducibility was based on the repeat assessment of all MR images 15 days after first evaluation.

\section{Results}

Of the 61 participants the average age was $40.1 \pm 14.3$ years; 44 were male and 17 were female; and the male/female ratio was 2.6. Fifty-nine of the bipartite fragments were located on the superolateral quadrant of the patella (Type III) and two were located on the lateral side (Type II).

Detailed MRI analysis showed oedema at the bipartite fragment or adjacent patella in 35 patients (57.4\%) and no oedema in 26 patients (42.6\%). In the evaluation of the radiological features of the synchondrosis, cartilage signal was detected in 26 patients (42.6\%), fibrous signal in six patients $(9.8 \%)$, and fluid signal in 29 patients (47.5\%). Table 1 presents descriptive statistics on the demographic and clinical characteristics of the cases. 
Accordingly, the average height of the fragment was $14.0 \mathrm{~mm}$, ranging from 8 to $34 \mathrm{~mm}$. The average distance between the fragment and the patella was $2.2 \mathrm{~mm}$ with a range of 0.6-5.6 $\mathrm{mm}$. Table 2 presents measurements of $\mathrm{FH}$ and FPD.

Among the patients with oedema across the bipartite area $(n=35) 25$ patients $(71.4 \%)$ had other major knee pathologies that can cause symptoms on the knee. Sixteen patients had no major MRI diagnosis, and 10 of these patients had oedema at bipartite fragment or adjacent patella (62.5\%). These 10 patients were assumed to be 'symptomatic' for bipartite patella, and the other 51 patients were assumed as 'asymptomatic' for bipartite patella. The results for group correlations regarding the findings among bipartite fragment are given in Table 3.

The age of the patients in the symptomatic bipartite patella group was statistically lower than in the asymptomatic group $(p<0.05)$. There was no statistically significant difference between the groups in terms of male and female distributions ( $p=0.257)$. There was no statistically significant difference in the incidence of cartilage, fibrous, and fluid signal for the asymptomatic and symptomatic groups ( $p>0.05)$. There was no statistically significant difference in FH and FPD measurements for the asymptomatic group and symptomatic groups, respectively $(p=0.907$ and $p=0.545)$.

\section{Bipartite patella prevalence}

In the last part of the study, 695 knee MRIs were assessed in order to evaluate the prevalence of bipartite patella, and the overall prevalence was found to be $0.8 \%$.

\section{Discussion}

Bipartite patella, which is rare and usually incidental, is a variant that can be diagnosed by various radiological methods and is thought to be of clinical importance [8]. MRI is a widely used tool for the assessment of the knee
Table 2. Measurements of fragment height $(\mathrm{FH})$ and distance between the fragment and the patella (FPD)

\begin{tabular}{|l|c|}
\hline & $n=61$ \\
\hline Average FH & $14.0 \mathrm{~mm}$ \\
\hline Range of FH & $8-34 \mathrm{~mm}$ \\
\hline Average FPD & $2.2 \mathrm{~mm}$ \\
\hline Range of FPD & $0.6-5.6$ \\
\hline
\end{tabular}

joint because of its advantages such as lack of radiation exposure, superior soft tissue resolution, and cartilage imaging priority. To the best of our knowledge, this is a unique report for the assessment of detailed morphological evaluation in patients with bipartite patella by MRI. The results of this study demonstrated that the prevalence of bipartite patella in the Turkish population is well correlated with the current literature $[9,10]$. Our study confirmed that bipartite patella occurs mainly in males, which has been previously well-documented [11].

Saupe classified bipartite patella into three types, depending on the localisation of the unfused fragment of patella [12]. Oohashi et al. recently proposed a new classification for developmental anomaly of the patella and added 'superolateral and lateral tripartite' and 'superolateral tripartite' patella to the new classification [13]. We used the tripartite or multipartite variant as an exclusion criterion in our study because we intended to evaluate the bipartite variant only. In our study, the highest rate was found on Type III (superolateral) variant, and the more rarely seen lateral localised fragment (Type II) was found only in two patients. Our results were evaluated as compatible with the most common and rare types defined in the literature by others $[6,13,14]$.

Moreover, symptomatology of this variant is still contradictory and is reported in $<2 \%$ of cases $[15,16]$. However, due to stress along the accessory synchondrosis, bipartite patella may be a cause of anterior knee pain, and it presents with bone marrow oedema around the bipartite

Table 3. Demographic and clinical characteristics regarding the groups

\begin{tabular}{|c|c|c|c|}
\hline & Asymptomatic $(n=51)$ & Symptomatic $(n=10)$ & $p$-value \\
\hline Age (years) $^{*}$ & $42.2 \pm 14.0$ & $29.5 \pm 11.3$ & $0.009^{\dagger}$ \\
\hline \multicolumn{4}{|l|}{ Gender } \\
\hline Male & $35(68.6 \%)$ & $9(90.0 \%)$ & \multirow[t]{2}{*}{$0.257^{\ddagger}$} \\
\hline Female & $16(31.4 \%)$ & $1(10.0 \%)$ & \\
\hline Cartilage signal & $23(45.1 \%)$ & $3(30.0 \%)$ & $0.494^{\ddagger}$ \\
\hline Fibrous signal & $7(13.7 \%)$ & $1(10.0 \%)$ & $>0.999^{\ddagger}$ \\
\hline Fluid signal & $21(41.2 \%)$ & $6(60.0 \%)$ & $0.315^{\ddagger}$ \\
\hline $\mathrm{FH}^{* *}$ & $13.0(11.0-16.0)$ & $14.0(11.0-15.1)$ & $0.907^{\natural}$ \\
\hline$F P D^{* *}$ & $2.0(1.4-2.3)$ & $2.2(1.6-3.4)$ & $0.545^{\natural}$ \\
\hline
\end{tabular}

*Data presented as mean $\pm S D,{ }^{* *}$ Descriptive statistics shown as median $\left(25^{\text {th }}-75^{\text {th }}\right)$ percentiles, ${ }^{\dagger}-$ Student's $t$-test, ${ }^{\ddagger}-$ Fisher's exact test, ${ }^{n}-$ Mann-Whitney $U$ test. 
fragment on MRI [17]. Radha et al. reported a case series with symptomatic bipartite patella, and all the patients had oedema across the synchondrosis as an MRI finding [18]. O'Brien et al. examined the knee MRIs of 25 asymptomatic patients with bipartite patella, and none of them had fragment oedema. Based on this result, they assumed that oedema accompanied by bipartite patella might be the cause of the symptoms, and the patients without oedema were asymptomatic [19]. In our study 10 of 61 patients had oedema as the only abnormal knee MRI finding, and this symptomatic (or oedema only) group was statistically younger than the asymptomatic group. Weckström et al. reported symptomatic patients who had undergone surgery for excision of a painful fragment; the median age at the time of surgery was 20 years [17]. Also, Ishikawa et al. reported the mean age of the patients with symptomatic bipartite patella as $15.7 \pm 4.4$ years [20]. Although these published reports defined patients with young age with symptomatic bipartite patella, our study is the first in the literature to identify statistical differences between age groups and symptomatology. As we mentioned previously, painful partite patella can be linked to microtraumas; decreasing age with increasing physical functioning may be the reason for this phenomenon. Another possible reason of this result may be that other painful knee pathologies that develop with age increase, and the pain caused by bipartite patella may be less considered.

Except for the defining symptomatology, MRI is an excellent examination for the evaluation of the signals of synchondrosis between the bipartite fragment and the main patellar bone because of the high resolution it provides. In the study performed by Ooashi et al., it was shown that histologically the interposed tissue between the bipartite fragment and the main patella can be fibrous tissue, fibrocartilage, or hyaline cartilage [21]. The results of this study - which was performed on excised patella specimens - constitute the foundation for the assessment of synchondrosis on MRI. MRI not only evaluates cartilage and fibrous signal very well, but also helps to detect fluid signal. In the evaluation of synchondrosis of the bipartite patella with MRI; Kavanagh et al. detected that overall $57 \%$ of patients had a hyaline cartilage signal of the patients with oedema within their bipartite fragment [10]. O'Brien et al. detected fluid signal in this region in $82 \%$ of 25 asymptomatic patients [19]. No association between signal characteristics of synchondrosis and oedema was shown in this study. We have interpreted these results as showing that the interposed signal has no association with the symptomatology.

Finally, MRI is a very good radiological method for the evaluation of the fragment morphology and for assessing the relationship between the fragment and the patella. A detailed evaluation of the fragment is important both in guiding the surgical approach in the treatment and in evaluating the relationship between the main patellar bone and the fragmented side [22]. In our study, we measured FH and FPD on MRI by using hypothetical reference points to provide standardisation for the measurements and intended confirmation of these reference points with two observers. The high rates we achieved in intra- and interobserver compliance suggest that our reference points may be a guide for future studies. Our study also contributes to the literature by describing the shortest/longest axis of the bipartite fragment and the narrowest/broadest measurements of the bipartite fragment-patella distance. In our study the distance between the bipartite fragment and main patella was an average of $2.2 \mathrm{~mm}$. Several authors reported mobility at the level of synchondrosis and excessive traction force by the vastus lateralis muscle on the bipartite fragment in symptomatic patients $[23,24]$. We measured the interface of synchondrosis by using FPD and found no relationship with the symptomatology. Thus, in our cases, separation of the bony interface does not contribute to pain. However, we believe that FPD should be evaluated with large symptomatic case groups in future studies, and it could become an important measurement when choosing between surgery or conservative treatment.

Our study also has limitations: firstly, even though we reached the largest patient series in the literature, the study was designed retrospectively. Secondly, because the exact symptoms and follow-up of the patients cannot be fully determined, it is questionable whether bipartite patella is the primary cause of the knee pain or not. Further research including detailed symptomatology of the patients and long-term follow-up is required to define the optimal MRI findings for this variant.

\section{Conclusions}

MRI is the most valuable diagnostic tool for the assessment of detailed morphological and pathological changes in patients with bipartite patella; it may additionally be valuable for the assessment of FH and FPD, which warrants further investigation. To our knowledge, a detailed MRI analysis like our study has not previously been performed, and our report is unique in showing that the symptomatic occurrence of bipartite patella is statistically higher in young patients. We think that this is a very valuable report for future MRI studies about this rare kneecap variant.

\section{Disclosure}

The authors declare no conflicts of interest. 
References

1. Gray DJ, Gardner E. Prenatal development of the human knee and superior tibiofibular joints. Am J Anat 1950; 86: 235-287.

2. Ogden JA. Radiology of postnatal skeletal development. X. Patella and tibial tuberosity. Skeletal Radiol 1984; 11: 246-257.

3. Saupe H. Primare Knochenmark seilerung der Kniescheibe. Dtsche Z Chir 1943; 258: 386.

4. McMahon SE, LeRoux JA, Smith TO, et al. The management of the painful bipartite patella: a systematic review. Knee Surg Sports Traumatol Arthrosc 2016; 24: 2798-2805.

5. Gruber W. In Bildungsanomalie mit Bildungshemmung begründete Bipartition beider Patellae eines jungen Subjectes. Archiv für Pathologische Anatomie und Physiologie und für Klinische Medicin 1883; 94: 358-361.

6. Oohashi Y, Koshino T, Oohashi Y. Clinical features and classification of bipartite or tripartite patella. Knee Surg Sports Traumatol Arthrosc 2010; 18: 1465-1469.

7. Weaver JK. Bipartite patellae as a cause of disability in the athlete. Am J Sports Med 1977; 5: 137-114.

8. Adachi N, Ochi M, Yamaguchi H, Uchio Y, Kuriwaka M. Vastus lateralis release for painful bipartite patella. Arthroscopy 2002; 18: 404-411.

9. Canizares G, Selesnick F. Bipartite patella fracture. Arthroscopy 2003; 19: 215-217.

10. Kavanagh EC, Zoga A, Omar I, et al. MRI findings in bipartite patella. Skeletal Radiol 2007; 36: 209-214.

11. Bourne MH, Bianco AJ. Bipartite patella in the adolescent: results of surgical excision. J Pediatr Orthop 1990; 10: 69-73.

12. Saupe E. Beitrag zur patella bipartita. Fortschr Rontgenstr 1921; 28: 37-41.
13. Oohashi Y. Developmental anomaly of ossification type patella partita. Knee Surg Sports Traumatol Arthrosc 2015; 23: 1071-1076.

14. Oohashi Y, Koshino T. Bone scintigraphy in patients with bipartite patella. Knee Surg Traumatol Arthrosc 2007; 15: 1395-1399.

15. Iossifidis A, Brueton RN. Painful bipartite patella following injury. Injury 1995; 26: 175-176.

16. Atesok K, Doral MN, Lowe J, et al. Symptomatic bipartite patella: treatment alternatives. J Am Acad Orthop Surg 2008; 16: 455-461.

17. Weckström M, Parviainen M, Pihlajamäki HK. Excision of painful bipartite patella. Good long-term outcome in young adults. Clin Orthop Relat Res 2008; 466: 2848-2855.

18. Radha S, Shenouda M, Konan S, et al. Successful treatment of painful synchondrosis of bipartite patella after direct trauma by operative fixation: a series of six cases. Open Orthop J 2017; 11: 390-396.

19. O’Brien J, Murphy C, Halpenny D, et al. Magnetic resonance imaging features of asymptomatic bipartite patella. Eur J Radiol 2011; 78: 425-429.

20. Ishikawa M, Adachi N, Deie M, et al. Unique patellofemoral alignment in a patient with a symptomatic bipartite patella. Knee 2016; 23: $127-132$.

21. Oohashi Y, Noriki S, Koshino T, et al. Histopathological abnormalities in painful bipartite patellae in adolescents. Knee 2006; 13: 189-193.

22. Canizares GH, Selesnick FH. Bipartite patella fracture. Arthroscopy 2003; 19: 215-217.

23. Ogata K. Painful bipartite patella. A new approach to operative treatment. J Bone Joint Surg Am 1994; 76: 573-578.

24. Carney J, Thompson D, O'Daniel J, Cassidy J. Arthroscopic excision of a painful bipartite patella fragment. Am J Orthop 2010; 39: 40-43. 